УДК 614.841.45

ТРЁХМЕРНОЕ МОДЕЛИРОВАНИЕ В ЗАДАЧАХ РАСЧЁТА И ВИЗУАЛИЗАЦИИ РАСПРОСТРАНЕНИЯ ОПАСНЫХ ФАКТОРОВ ПОЖАРА ПРИ ОЦЕНКЕ ПОЖАРНЫХ РИСКОВ

\title{
THREE-DIMENSIONAL SIMULATION FOR CALCULATION AND VISUALIZATION OF THE DANGEROUS FIRE FACTOR PROPAGATION IN THE FIRE RISK ASSESSMENT
}

\author{
Р.А. Валиуллина, Л.Р. Аминева, А.А. Шарафутдинов
}

Уфимский государственный нефтяной технический университет, г. Уфа, Российская Федерация

\author{
Regina A. Valiullina, Lilya R. Amineva, Azat A. Sharafutdinov \\ Ufa State Petroleum Technological University, \\ Ufa, Russian Federation \\ e-mail: valiregaz03@gmail.com
}

Аннотация. Важной составляющей условий соответствия объекта защиты требованиям пожарной безопасности является расчет пожарного риска, который подразумевает нахождение величины, определяющей частоту воздействия опасных факторов пожара на человека, находящегося в здании.

Немаловажным аспектом при оценке пожарных рисков являются расчет и визуализация распространения опасных факторов пожара. Для описания развития параметров состояния среды в помещении в течение определенного времени, а также изменения параметров состояния ограждающих конструкций используется трехмерное моделирование. Цель работы - выявить необходимость применения 3D моделирования в задачах 
расчета и визуализации опасных факторов пожара при оценке пожарных рисков.

В настоящей статье изучены теоретические основы применения трехмерного моделирования при расчете пожарных рисков. Выявлены особенности и проблемы использования 3D модели для визуализации опасных факторов пожара. Подробно описан процесс определения расчетной величины пожарного риска с применением трехмерного моделирования. Рассмотрены преимущества и недостатки применения 3D моделирования.

В ходе проведенного исследования выявлено, что применение трехмерного моделирования в задачах расчета и визуализации опасных факторов пожара при оценке пожарных рисков является целесообразным, актуальным, в несколько раз упрощает решение поставленных задач.

Abstract. The important component of the conditions for the target protected asset to satisfy the fire safety requirements is calculation of fire risk, i.e., it is required to obtain the value determining frequency of fire hazards exposure for a person inside the affected building.

An important aspect in the assessment of fire risks is calculation and visualization of fire hazards propagation. Three-dimensional modeling is used for describing the development of parameters of the room environment condition over time and for describing the changes in the parameters of the building envelope condition. The purpose of the work is determination of the necessity for using 3D modeling in calculation and visualization of fire hazards within the scope of fire risks assessment.

This article studies theoretical foundations of the application of threedimensional modeling in fire risks calculation. The specific features and problems of using a 3D model for visualizing fire hazards have been identified. The process of determination of the fire risk rated value with application of three-dimensional modeling is described in detail. The author has reviewed advantages and disadvantages of 3D modeling. 
It was discovered in the course of the study that the use of three-dimensional modeling in resolving the tasks of calculation and visualization of fire hazards within the scope of assessment of fire risks is appropriate, relevant, and it simplifies several fold the solution of the set tasks.

Ключевые слова: пожарный риск; опасные факторы пожара; трехмерное моделирование; 3D модель; критические значения; вероятность эвакуации; модель пожара

Keywords: fire risk; fire hazards; three-dimensional modeling; 3D model; critical values; evacuation probability; fire model

С вступлением в силу технического регламента [1] изменился общий подход к нормативным положениям в области пожарной безопасности, появилась возможность гибкого нормирования или выбора условий соответствия обеспечения пожарной безопасности: расчет пожарного риска, либо выполнение требований нормативных документов [2].

В данной статье рассмотрена методика определения расчетной величины пожарного риска, которая указана в приказе МЧС № 382 [3].

Немаловажными являются расчет и визуализация распространения опасных факторов пожара (ОФП). К опасным факторам пожара относят факторы, влияние которых может привести к травме человека, его отправлению, летальному исходу, а также материальному ущербу имущества.

Технический регламент [1] определяет перечень ОФП, подразделяет их на две категории. Определяют опасные факторы пожара, которые способны оказывать воздействие на людей и их материальные ценности: огонь и искры, количество тепла, которое проходит через поверхность в единицу времени, воздействие высокой температуры, увеличение концентрации продуктов горения, уменьшение концентрации кислорода, потеря видимости в дыму. 
Осколки, части разрушившегося оборудования, сооружений; токсичные вещества, которые попали в окружающую среду из технологических аппаратов; неправильное использование огнетушащих веществ и материалов, высокое напряжение, возникшее на токопроводящих частях оборудования, конструкций, факторы взрыва, которые возникают вследствие пожара, относятся к сопутствующим проявлениям опасных факторов пожара.

В таблице 1 приведены существующие критические значения по каждому из опасных факторов пожара [3].

Таблица 1. Критические значения опасных факторов пожара

\begin{tabular}{|c|c|}
\hline Опасный фактор пожара & Предельное значение \\
\hline Содержание окиси углерода СО & $1,16 \Gamma / \mathrm{M}^{3}$ \\
\hline Содержание двуокиси углерода $\mathrm{CO}_{2}$ & $0,00011 \Gamma / \mathrm{M}^{3}$ \\
\hline Содержание хлористого водорода $\mathrm{HCl}$ & $0,000023 \Gamma / \mathrm{M}^{3}$ \\
\hline Температура & $+70{ }^{\circ} \mathrm{C}$ \\
\hline Тепловой поток & $1400 \mathrm{BT} / \mathrm{M}^{2}$ \\
\hline Предельная видимость в дыму & $20 \mathrm{M}$ \\
\hline
\end{tabular}

Для проведения расчета величины пожарного риска необходимо определение исходных данных: конструктивных и объемно-планировочных параметров здания, теплофизических свойств ограждающих конструкций и размещенных в здании аппаратов и оборудования, горючие материалы, их расположение, количество людей и место их сосредоточения в здании, наличие АУПС, АУПТ, СОУЭ, а также систем противодымной защиты [4-14].

После чего анализируется пожарная опасность здания с применением перечня исходных данных. Вместе с тем учитывается динамика развития пожара и результат воздействия опасных факторов пожара на людей, которые находятся в здании [3]. 
Приказ МЧС № 382 [3] также определяет необходимость выбора сценария развития пожара. Сценарий пожара - это вариант его развития, при котором учитывается место возникновение возгорания, характер его развития. Сценарий развития пожара формируется на основании конструктивных решений, размещении пожарной нагрузки и количества людей на объекте. Хотелось бы отметить, что рассматривается сценарий, при котором наблюдаются наихудшие последствия для обеспечения безопасности людей.

В качестве наихудших условий, как правило, выступают помещения с массовым пребыванием людей, атриумного типа. Порядок условий определен в приказе МЧС [3].

Для оценки динамики развития опасных факторов пожара при расчете пожарных рисков используется трехмерное моделирование.

С целью построения полей ОФП необходимо использование соответствующего программного обеспечения, которое позволит выполнять расчет пожарного риска с высокой точностью. Компоненты, используемые в программах для расчета пожарного риска с разработкой трехмерной модели, позволяют упростить выполнение задач:

1. построитель трехмерного каркаса здания, расчетных областей для моделирования пожара и эвакуации людей;

2. возможность редактирования сценариев пожара;

3. модуль, который реализует расчет развития пожара различными математическими моделями;

4. модуль для трехмерной визуализации опасных факторов пожара;

5. модуль, определяющий расчетную величину пожарного риска $[5,12]$.

Применение трехмерного моделирования имеет ряд преимуществ.

Во-первых, работа ведется в единой программной среде, что способствует комфортному решению задач расчета движения людей и распространения опасных факторов пожара. 
Во-вторых, существует возможность использования поэтажных планов по подложке, импортировать любую модель здания в программное обеспечение.

В-третьих, трехмерная виртуальная среда объекта позволяет изменять позицию наблюдателя.

Используемый встроенный модуль 3D визуализации позволяет в разных частях здания прослеживать процесс эвакуации людского потока и распространения полей опасных факторов пожара: теплового потока, температуры окружающей среды, концентраций токсичных продуктов горения, видимости в дыму.

Пространственная визуализация, возможная при применении трехмерного моделирования, позволяет наглядно проследить динамику развития пожара, оценивать влияние риска образования факторов на исход эвакуации людей.

Программное обеспечение, которое используется при построении полей опасных факторов пожара, позволяет выполнить постановку задачи и моделирование развития пожара в соответствии с предъявляемыми требованиями [3].

В первую очередь, необходимо создание трехмерного каркаса, который включает в себя все значимые элементы для сценария.

На рисунке 1 показано создание трехмерного каркаса здания.

Возможно использование графических изображений поэтажных планов этажей. Затем на основе каркаса необходимо создание расчетной области для моделирования развития пожара. Для выполнения этой задачи осуществляется присоединение всех значимых для сценария развития пожара этажей здания, лестничных клеток, проемов. 


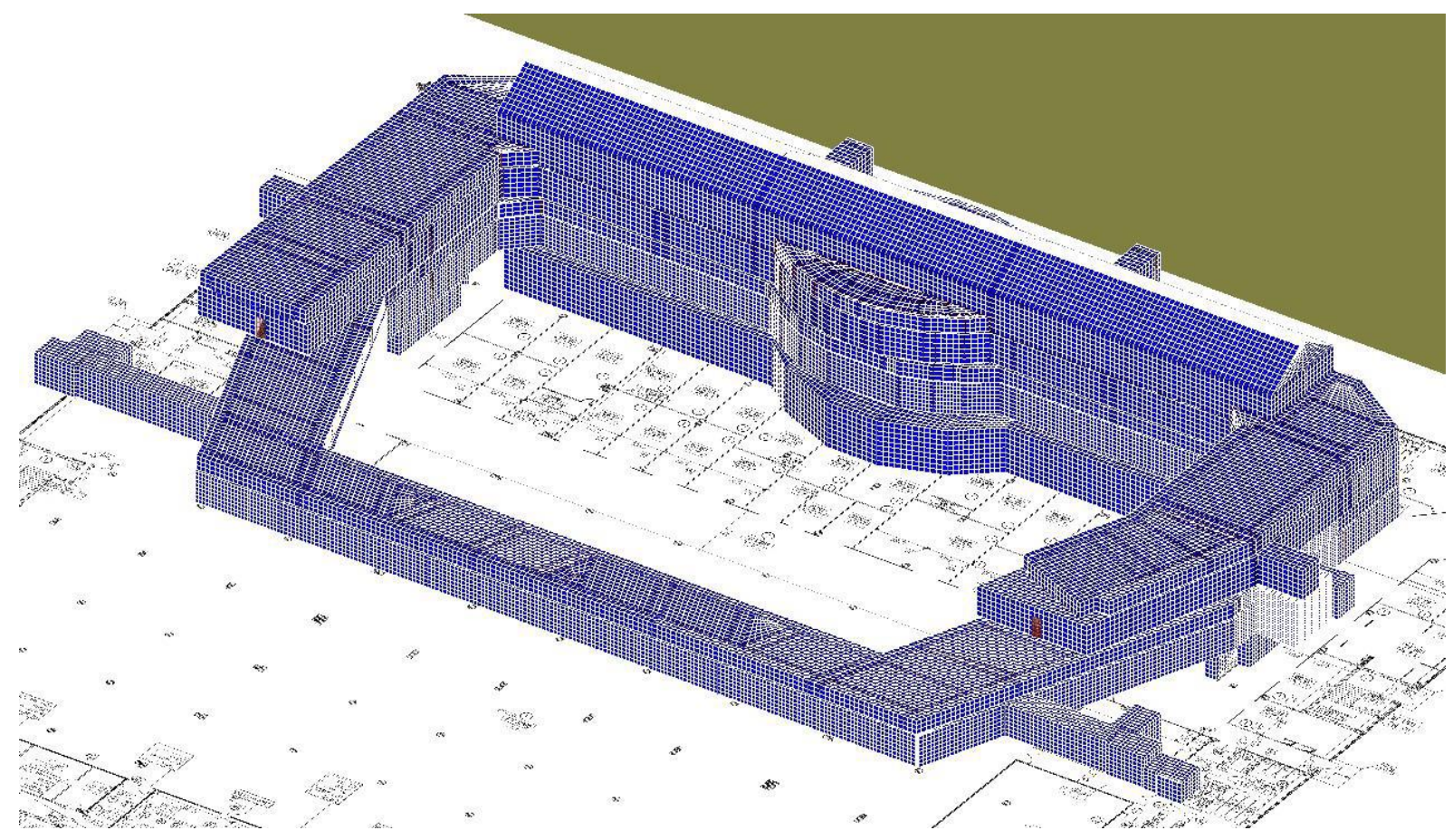

Рисунок 1. Трехмерный каркас здания

Необходимо указание области расположения пожарной нагрузки. Задаются окна в помещении для того, чтобы учесть тепловые потери, выходы наружу из здания. Также необходимо задать шаг сетки в расчетной области и ее построение. Это все необходимо для визуализации сетки и связей между смежными блоками $[5,13]$.

После чего на основе каркаса создается расчетная область для моделирования эвакуации людей. Для выполнения данного этапа необходимо включить все значимые для сценария объемы этажей здания и лестничных клеток, проемы, выходы наружу из здания.

После проведения подготовительных этапов необходимо ввести начальные данные для моделирования динамики развития опасных факторов пожара. Задаются условия: начальная температура воздуха в расчетной области, температура воздуха в окружающей среде, пожарная нагрузка, находящаяся в расчетной области, координаты очага развития пожара. Также необходимы данные о количестве проемов, дверей, окон, отверстий для вытяжной и приточной противодымной вентиляций с описанием массовых расходов через них. Немаловажным является указание стен, поскольку при расчете учитывается их прогрев. 
Осуществляется ввод данных для непосредственно моделирования эвакуации.

Для этого производится расстановка людей на этажах здания. Необходимо задать индивидуально и группам людей соответствующие характеристики: время начала эвакуации, путь, площадь проекции, начальную скорость движения, группу мобильности. В помещениях расставляется пожарная нагрузка, препятствия для движения людского потока.

При помощи программного обеспечения выполняется расчет развития пожара и после его завершения создаются горизонтальные сечения полей опасных факторов пожара в заданной области до последующей визуализации и совместного анализа результатов расчетов распространения ОФП и эвакуации.

На рисунке 2 изображено поле опасных факторов пожара при блокировании путей эвакуации по потере видимости.


Frame: 1599

Рисунок 2. Блокирование путей эвакуации по потере видимости

После расчета развития пожара проводится расчет эвакуации, затем выполняется совместная визуализация полей опасных факторов пожара на 
каждом этапе здания и эвакуирующихся людей. При этом доступно отображения полей ОФП: видимость, тепловой поток, концентрация токсичных продуктов горения, плотность потока людей.

Затем выполняется анализ результатов расчета сценария и определение вероятности эвакуации людей. Проводится совместный анализ процесса эвакуации и развития пожара, в которой отображены данные по началу эвакуации, её длительности, окончанию, времени блокирования ОФП, длительность скопления, а также вероятности эвакуации.

После вышеперечисленных действий становится возможным определение величины пожарного риска.

В настоящий момент методика расчета пожарного риска основана по большей части на двумерном моделировании, которое не всегда наглядно и информативно показывает развитие динамики пожара и распространение опасных факторов пожара.

Однако 3D моделирование получило широкое распространение в различных отраслях деятельности человека, в том числе и при расчете пожарного риска.

Авторы статьи [6] считают, что трёхмерная модель помещения позволит более оптимально выполнить расчет распространения опасных факторов пожара. В статьях [6, 15-18] приводятся примеры использования компьютерного программного комплекса «СИГМА ПБ», предназначенного для выполнения расчетов ОФП и эвакуации из зданий и сооружений различного назначения с использованием 3D моделирования.

В работе [7] С.А. Донец и С.М. Санникова отмечают, что применение 3D моделирования позволит проанализировать пространственную взаимосвязь между всеми элементами системы объекта в совокупности с данными. Преимуществом 3D моделирования, по мнению авторов, являются автоматический контроль и мониторинг подконтрольных объектов, более наглядное представление различных вариантов распространения пожара. 
В статьях $[6,7,16]$ очевидными преимуществами 3D моделирования при расчетах пожарных рисков являются наглядность, информативность и точность.

Не подлежит сомнению тот факт, что 3D моделирование необходимо внедрять в методику пожарного риска с целью повышения точности, более оптимального выполнения расчета.

При проведении расчета пожарного риска возникает достаточное количество проблем.

В работе [8] автор рассматривает возникновение важных вопросов при оценке пожарных рисков.

В некоторых случаях расчет необходимого времени для эвакуации людей, выполненный по методикам, не согласовывается с данными проведенных экспериментальных исследований, полученными в ходе анализа протекания реальных пожаров.

Расхождения экспериментальных и натурных исследований в ходе анализа протекания возгораний объясняются, в первую очередь, тем, что проблема математического моделирования динамики развития опасных факторов пожара во время эвакуации людей должным образом не решена. При разработке проектной документации с учетом требований пожарной безопасности к путям эвакуации достаточно часто расчет ОФП не принимается во внимание $[8,17,19]$.

Автор [8] отмечает, что «точность расчета динамики ОФП в большой степени определяется достоверностью исходных данных по свойствам пожарной нагрузки. Определение величины удельной массовой скорости выгорания твердых горючих веществ и жидкости в еще неустановившемся процессе горения это одна из наименее изученных проблем».

Е.А. Миклина, и С.Н. Волкова в статье о проблемах моделирования динамики пожара [9] также рассматривают вопрос о существующих проблемах моделирования при расчетах пожарного риска. Авторы утверждают, что актуализирован ряд проблем, допущений и упрощений, 
которые увеличивают погрешность расчетов и ограничивают область применения используемых математических моделей пожара.

Имеются следующие принципиальные проблемы: множество физических процессов требуют выполнение детального моделирования, вследствие чего возникает необходимость в разработке сложных численных алгоритмов, которые позволяют адаптировать модель к конкретным условиям задачи. Для реализации полевых математических моделей пожара необходимы вычислительные и временные затраты. Вследствие этого ограничен размер системы помещений [10, 20].

\section{Выводы}

Несмотря на недостатки (трудоёмкость выполнения работы, возможные денежные затраты на программное обеспечение) трехмерное моделирование - это, несомненно, технологичное, перспективное и актуальное решение, которое позволяет находить ответы на поставленные задачи точно и оперативно.

\section{Список используемых источников}

1. Федеральный закон от 22.07.2008 № 123-Ф3 «Технический регламент о требованиях пожарной безопасности» (с посл. изм. и доп. от 30.04.2021) // Информационно-правовое обеспечение «Гарант». URL: https://base.garant.ru/12161584/ (дата обращения: 21.06.2021).

2. Приказ МЧС РФ от 10.07.2009 № 404 «Об утверждении методики определения расчетных величин пожарного риска на производственных объектах» (с посл. изм. и доп. от 14.12.2010) // Информационно-правовое обеспечение «Гарант». URL: https://base.garant.ru/196118/ (дата обращения: 21.06.2021). 
3. Приказ МЧС РФ от 30.06.2009 № 382 «Об утверждении методики определения расчетных величин пожарного риска в зданиях, сооружениях и строениях различных классов функциональной пожарной опасности» (с посл. изм. и доп. от 02.12.2015) // Информационно-правовое обеспечение «Гарант». URL: https://base.garant.ru/12161584/ (дата обращения: 21.06.2021).

4. Брушлинский Н.Н., Клепко Е.А. К вопросу о вычислении рисков // Проблемы безопасности и чрезвычайных ситуаций. 2004. № 1. С. 71-73.

5. Официальный сайт Сигма ПБ. URL: https://3ksigma.ru/soft/ (дата обращения: 24.06.2021).

6. Чернодуб С.C., Беседина С.В. Роль и значение 3D моделирования в системе МЧС // Современные технологии обеспечения гражданской обороны и ликвидации последствий чрезвычайных ситуаций. 2015. № 1-1 (6). С. 207-209.

7. Донец С.A., Санникова С.M. Применение 3D визуализации для проведения пожарно-тактических расчетов с учетом современных информационных технологий // Пожарная безопасность: проблемы и перспективы. 2017. Т. 1. № 8 (607). С. 58-60.

8. Колодяжный С.А. Прогнозирование времени блокирования путей эвакуации опасными факторами пожара в многофункциональных центрах: дис. ... д-ра. техн. наук. Воронеж: Изд-во ВГТУ, 2017. 269 с.

9. Миклина Е.А., Волкова С.Н. О проблемах моделирования динамики пожара // Пожарная безопасность: проблемы и перспективы. 2018. Т. 1. № 9. C. 599-602.

10. Колодяжный С.А., Переславцева И.И. Математическое моделирование динамики основных опасных факторов в начальной стадии пожара // Известия Казанского государственного архитектурностроительного университета. 2014. № 4 (30). С. 403-412.

11. Программа для ЭВМ 2018610396 РФ. Программа для расчета параметров пожаров и аварийных розливов нефти / Р.А. Исмаков, Ф.Ш. Хафизов, И.Ф. Хафизов, А.А. Шарафутдинов, Р.Р. Курбанаев, Р.Р. Каримов. 2017661649, Заявлено 14.11.2017; Опубл. 10.01.2018. 
12. Программа для ЭВМ 2018610351 РФ. Программа моделирования и расчета водопенных коммуникаций пожарных автоцистерн / Р.Н. Бахтизин, О.А. Баулин, Ф.Ш. Хафизов, И.Ф. Хафизов, А.А. Шарафутдинов, А.Т. Шайдуллин. 2017661738, Заявлено 14.11.2017; Опубл. 10.01.2018.

13. Программа для ЭВМ 2021610493 РФ. Программа для визуализации и получения аналитического решения многокритериальных задач / Р.Р. Валиев, О.А. Баулин, Ф.Ш. Хафизов, И.Ф. Хафизов, А.А. Альмухаметов, Аз.А. Шарафутдинов, А.А. Шарафутдинова. 2020667704 , Заявлено 25.12.2020; Опубл. 14.01.2021.

14. Gazizov A.M., Kuznetsova O.V., Sharafutdinov A.A., Shaimuhametova K.M. Improvement of Fire Retardant Properties of Wood Materials // IOP Conference Series: Materials Science and Engineering. 2020. Vol. 919. Article Number 062014. DOI: 10.1088/1757-899X/919/6/062014.

15. Sharafutdinov A.A., Khafizov F.S., Khafizov I.F., Krasnov A.V., Akhmethafizov A.V., Zakirova V.I., Khafizova A.N. Development of a Method for Calculating Fire and Oil Spills Parameters // AIP Conference Proceedings. 2020. Vol. 2216. Issue 1. Article Number 070004. DOI: 10.1063/5.0003843.

16. Sharafutdinov A.A., Timasheva A.Y. Structural and Intelligent Scheme of Navigation System of a Ground-Based Mobile Robot for Forming a Traffic Route // IOP Conference Series: Materials Science and Engineering. 2020. Vol. 860. Article Number 012019. DOI: 10.1088/1757-899X/860/1/012019.

17. Khafizov F.S., Gazizov A.M., Khafizov I.F., Sharafutdinov A.A. Evaluation of the Mobile Simulator for Fire Protection Training // Computer Systems, Applications And Software Engineering, CSASE 2018: Proceedings Annual Scientific International Conference. Nizhniy Tagil, Russia. 2018.

18. Устюжанина А.Ю., Ганиева А.А., Шарафутдинов А.А. Прогнозирование чрезвычайных ситуаций на предприятиях нефтехимии с применением геоинформационных технологий // Современные технологии в нефтегазовом деле - 2016: сб. тр. международ. науч.-технич. конф. посвященной 60-летию филиала. Уфа: Изд-во УГНТУ, 2016. С. 442-447. 
19. Хафизов И.Ф., Кудрявцев А.А., Шевченко Д.И., Шарафутдинов А.А. Проектирование технических средств обучения для специалистов нефтегазового комплекса на основе оптимального множества тренигов // Современные технологии в нефтегазовом деле - 2016: сб. тр. международ. науч.-технич. конф. посвященной 60-летию филиала. Уфа: Изд-во УГНТУ, 2016. C. 366-369.

20. Шарафутдинов А.А., Пономарева Е.А., Егорова Е.С. Особенности применения информационно-ситуационных технологий в области обеспечения комплексной безопасности объектов // Проблемы обеспечения безопасности при ликвидации последствий чрезвычайных ситуаций. 2016. № 1-2 (5). С. 194-196.

\section{References}

1. Federal'nyi zakon ot 22.07.2008 № 123-FZ «Tekhnicheskii reglament o trebovaniyakh pozharnoi bezopasnosti» (s posl. izm. i dop. ot 30.04.2021 g.) [RF Federal Law of July 22, 2008 No. 123-FZ «Technical Regulations on Safety Requirements» (with the Additions and Amendments of April 30, 2021)]. Informatsionno-pravovoe obespechenie «Garant». Available at: https://base.garant.ru/12161584/ (accessed 21.06.2021). [in Russian].

2. Prikaz MChS RF ot 10.07.2009 № 404 «Ob utverzhdenii metodiki opredeleniya raschetnykh velichin pozharnogo riska na proizvodstvennykh ob"ektakh» (s posl. izm. i dop. ot 14.12.2010 g.) [Order of the EMERCOM of the Russian Federation of July 10, 2009 No. 404 «On Approval of the Methodology for Determining the Calculated Values of Fire Risk at Production Facilities» (with the Additions and Amendments of December 14, 2010)]. Informatsionnopravovoe obespechenie «Garant». Available at: https://base.garant.ru/196118/ (accessed 21.06.2021). [in Russian]. 
3. Prikaz MChS RF ot 30.06.2009 № 382 «Ob utverzhdenii metodiki opredeleniya raschetnykh velichin pozharnogo riska $\mathrm{v}$ zdaniyakh, sooruzheniyakh i stroeniyakh razlichnykh klassov funktsional'noi pozharnoi opasnosti» (s posl. izm. i dop. ot 02.12.2015 g.) [Order of the EMERCOM of the Russian Federation of June 30, 2009 No. 382 «On Approval of the Methodology for Determining the Calculated Values of Fire Risk in Buildings, Structures and Structures of Various Classes of Functional Fire Hazard» (with the Additions and Amendments of December 2, 2015)]. Informatsionno-pravovoe obespechenie «Garant». Available at: https://base.garant.ru/12161584/ (accessed 21.06.2021). [in Russian].

4. Brushlinskii N.N., Klepko E.A. K voprosu o vychislenii riskov [On Calculating Risks]. Problemy bezopasnosti i chrezvychainykh situatsii - Safety and Emergencies Problems, 2004, No. 1, pp. 71-73. [in Russian].

5. Official Website of Sigma PB. Available at: https://3ksigma.ru/soft/ (accessed 24.06.2021). [in Russian].

6. Chernodub S.S., Besedina S.V. Rol' i znachenie 3D modelirovaniya $v$ sisteme MChS [The Role and Importance of 3D Modeling in the EMERCOM of Russian]. Sovremennye tekhnologii obespecheniya grazhdanskoi oborony i likvidatsii posledstvii chrezvychainykh situatsii - Modern Technologies for Civil Defense and Emergency Response, 2015, No. 1-1 (6), pp. 207-209. [in Russian].

7. Donets S.A., Sannikova S.M. Primenenie 3D vizualizatsii dlya provedeniya pozharno-takticheskikh raschetov s uchetom sovremennykh informatsionnykh tekhnologii [The Use of 3D Visualization for Conducting Fire-Tactical Calculations, Taking into Account Modern Information Technologies] Pozharnaya bezopasnost': problemy i perspektivy - Fire Safety: Problems and Prospects, 2017, Vol. 1, No. 8 (607), pp. 58-60. [in Russian].

8. Kolodyazhnyi S.A. Prognozirovanie vremeni blokirovaniya putei evakuatsii opasnymi faktorami pozhara $v$ mnogofunktsional'nykh tsentrakh: dis. d-ra. tekhn. nauk [Predicting the Time of Blocking Escape Routes by Hazardous Fire Factors in Multifunctional Centers: Doct. Engin. Sci. Diss.]. Voronezh, VGTU Publ., 2017. 269 p. [in Russian]. 
9. Miklina E.A., Volkova S.N. O problemakh modelirovaniya dinamiki pozhara [On the Problems of Modeling the Dynamics of Fire]. Pozharnaya bezopasnost': problemy i perspektivy - Fire Safety: Problems and Prospects, 2018, Vol. 1, No. 9, pp. 599-602. [in Russian].

10. Kolodyazhnyi S.A., Pereslavtseva I.I. Matematicheskoe modelirovanie dinamiki osnovnykh opasnykh faktorov v nachal'noi stadii pozhara [Mathematical Modeling of the Dynamics of the Main Hazards in the Initial Stage of Fire]. Izvestiya Kazanskogo gosudarstvennogo arkhitekturno-stroitel'nogo universiteta - News of the Kazan State University of Architecture and Engineering, 2014, No. 4 (30), pp. 403-412. [in Russian].

11. Ismakov R.A., Khafizov F.Sh., Khafizov I.F., Sharafutdinov A.A., Kurbanaev R.R., Karimov R.R. Programma dlya rascheta parametrov pozharov i avariinykh rozlivov nefti [Program for Calculating the Parameters of Fires and Emergency Oil Spills]. Computer Program RF, No. 2018610396, 2018. [in Russian].

12. Bakhtizin R.N., Baulin O.A., Khafizov F.Sh., Khafizov I.F., Sharafutdinov A.A., Shaidullin A.T. Programma modelirovaniya i rascheta vodopennykh kommunikatsii pozharnykh avtotsistern [Program for Modeling and Calculating Water-Foam Communications for Fire-Fighting Tankers]. Computer Program RF, No. 2018610351, 2018. [in Russian].

13. Valiev R.R., Baulin O.A., Khafizov F.Sh., Khafizov I.F., Almukhametov A.A., Sharafutdinov Az.A., Sharafutdinova A.A. Programma dlya vizualizatsii i polucheniya analiticheskogo resheniya mnogokriterial'nykh zadach [A Program for Visualization and Obtaining Analytical Solutions for Multicriteria Problems]. Computer Program RF, No. 2021610493, 2021. [in Russian].

14. Gazizov A.M., Kuznetsova O.V., Sharafutdinov A.A., Shaimuhametova K.M. Improvement of Fire Retardant Properties of Wood Materials. IOP Conference Series: Materials Science and Engineering, 2020, Vol. 919, Article Number 062014. DOI: 10.1088/1757-899X/919/6/062014. 
15. Sharafutdinov A.A., Khafizov F.S., Khafizov I.F., Krasnov A.V., Akhmethafizov A.V., Zakirova V.I., Khafizova A.N. Development of a Method for Calculating Fire and Oil Spills Parameters. AIP Conference Proceedings, 2020, Vol. 2216, Issue 1, Article Number 070004. DOI: 10.1063/5.0003843.

16. Sharafutdinov A.A., Timasheva A.Y. Structural and Intelligent Scheme of Navigation System of a Ground-Based Mobile Robot for Forming a Traffic Route. IOP Conference Series: Materials Science and Engineering, 2020, Vol. 860, Article Number 012019. DOI: 10.1088/1757-899X/860/1/012019.

17. Khafizov F.S., Gazizov A.M., Khafizov I.F., Sharafutdinov A.A. Evaluation of the Mobile Simulator for Fire Protection Training. Proceedings Annual Scientific International Conference «Computer Systems, Applications and Software Engineering, CSASE 2018». Nizhniy Tagil, Russia. 2018.

18. Ustyuzhanina A.Yu., Ganieva A.A., Sharafutdinov A.A. Prognozirovanie chrezvychainykh situatsii na predpriyatiyakh neftekhimii $\mathrm{s}$ primeneniem geoinformatsionnykh tekhnologii [Prediction of Emergency for Petrochemical Plants Using Information Technology]. Sbornik trudov Mezhdunarodnoi nauchno-tekhnicheskoi konferentsii posvyashchennoi 60-letiyu filiala «Sovremennye tekhnologii v neftegazovom dele - 2016» [Proceedings of the International Scientific and Technical Conference Dedicated to the 60th Anniversary of the Branch «Modern Technologies in Oil and Gas Business 2016»]. Ufa, UGNTU Publ., 2016, pp. 442-447. [in Russian].

19. Khafizov I.F., Kudryavtsev A.A., Shevchenko D.I., Sharafutdinov A.A. Proektirovanie tekhnicheskikh sredstv obucheniya dlya spetsialistov neftegazovogo kompleksa na osnove optimal'nogo mnozhestva trenigov [Designing Technical Training for Specialists of Oil and Gas Complex on the Basis of the Optimal Number of Trainings]. Sbornik trudov Mezhdunarodnoi nauchno-tekhnicheskoi konferentsii posvyashchennoi 60-letiyu filiala «Sovremennye tekhnologii v neftegazovom dele 2016» [Proceedings of the International Scientific and Technical Conference Dedicated to the 60th Anniversary of the Branch «Modern Technologies in Oil and Gas Business - 2016»]. Ufa, UGNTU Publ., 2016, pp. 366-369. [in Russian]. 
20. Sharafutdinov A.A., Ponomareva E.A., Egorova E.S. Osobennosti primeneniya informatsionno-situatsionnykh tekhnologii $\mathrm{v}$ oblasti obespecheniya kompleksnoi bezopasnosti ob"ektov [Features of the Use of Information and Situational Technologies in the Field of Ensuring the Integrated Security of Facilities]. Problemy obespecheniya bezopasnosti pri likvidatsii posledstvii chrezvychainykh situatsii - Safety Issues in Disaster Management, 2016, No. 1-2 (5), pp. 194-196. [in Russian].

\section{Сведения об авторах}

\section{About the authors}

Валиуллина Регина Азаматовна, студент кафедры ««Пожарная и промышленная безопасность», УГНТУ, г. Уфа, Российская Федерация

Regina A. Valiullina, Student of Fire and Industrial Safety Department, USPTU, Ufa, Russian Federation

e-mail: valiregaz03@gmail.com

Аминева Лилия Рустамовна, студент кафедры ««Пожарная и промышленная безопасность», УГНТУ, г. Уфа, Российская Федерация

Lilya R. Amineva, Student of Fire and Industrial Safety Department, USPTU, Ufa, Russian Federation

e-mail: aminevalr99@mail.ru

Шарафутдинов Азат Амирзагитович, канд. техн. наук, доцент кафедры «Пожарная и промышленная безопасность», УГНТУ, г. Уфа, Российская Федерация

Azat A. Sharafutdinov, Candidate of Engineering Sciences, Assistant Professor of Fire and Industrial Safety Department, USPTU, Ufa, Russian Federation

e-mail: azat_sharaf@mail.ru 